\title{
Convergent validity of the Richmond Reversal Rating in relation to visual- spatial perception as measured by the SASP
}

\author{
Lucinda Venter, BSc OT (Hons) (ECU)
}

Janet Richmond, BOT (Hons) (UP), MOT (UKZN), PhD (ECU)

Senior Lecturer, School of Medical and Health Sciences, Edith Cowan University, Australia

\author{
Myra F Taylor, MPhil, PhD \\ Research Fellow, Edith Cowan University, Australia
}

This study examined the convergent validity of the visual perceptual Richmond Reversal Rating (RRR) assessment, in relation to visualspatial abilities of young school-aged children, using known-groups validity and convergent validity hypotheses testing. Seventy-two primary school children (Years I-3) were assessed with the RRR assessment and the Spatial Awareness Skills Program Test (SASP). The Kruskal-Wallis test demonstrated a significant difference between the RRR overall scores and the SAPS grouped scores, $H(2)=6.155$, $p=.046$. Spearman's correlation coefficient revealed a low positive yet significant correlation $\left(r_{\mathrm{s}}=.433, p=.000\right)$ between the RRR overall scores and the SASP percentile scores, and a significant moderate positive correlation between the RRR overall scores and the SASP scores $\left(r_{s}=.666, p=.000\right)$. The results provide evidential support for the convergent validity of the RRR assessment in relation to visual-spatial abilities as measured by the SASP.

Key words: Letter reversals, number reversals, visual-spatial abilities, school based occupational therapy

\section{INTRODUCTION}

Since the twentieth century, children's visual motor and visual-spatial perceptual reading and writing errors have been a phenomenon of ongoing interest ${ }^{1-10}$. Children who have difficulty identifying the accurate orientation of numbers and letters often perform at a lower academic level, have lower reading abilities, and have less legible handwriting than their peers ${ }^{10-15}$. While boys have been documented as having three times the rate of visual perceptual difficulties as girls, recent research has questioned this gender difference suggesting that reversal errors in girls are often overlooked ${ }^{16,17}$.

Identifying boys and girls at risk of experiencing increased levels of academic difficulties is not only important for their academic functioning but for general functioning ${ }^{8,18}$. Studies have determined that older children and adults with academic and learning difficulties are more prone to behavioural difficulties ${ }^{19}$, educational dropout, suicide $^{20}$ and anxiety ${ }^{21,22}$.

Teachers have long identified legibility as an essential criterion when evaluating children's handwriting abilities ${ }^{23}$. In this regard, visual perceptual skills (e.g., visual discrimination and visual-spatial skills) have been identified as important cognitive skills that children need in order to write legibly (i.e., constructing letters with the correct form, consistent sizing, positioning and directional orientation ${ }^{14}$. Visual perception comprises a number of subtypes, including (but not limited to), visual discrimination and visual-spatial skills. Visual perception is the ability to conceptually assimilate, understand and interpret the visual sensory stimulus presented and to develop meaning from this information for use in verbal or physical output ${ }^{24}$. Visual discrimination is a component of visual perception which enables the person to expedite the differentiation of similarities and differences of visual input ${ }^{25-27}$. Visual discrimination precedes the ability to differentiate changes in direction which, in turn, assist visual-spatial skills ${ }^{27}$. Visual-spatial skills enable children to perceive the position of objects related to their own body/or other objects, such as linking the direction of an ' $r$ ' in relation to the line on the paper $^{26,28,29}$. At the stage when children are entering school, visual perception is at the optimum period of development (four and a half to seven years), but may continue up to 12 years of age. It is thus at this stage that it is important to identify and address potential difficulties in visual perceptual skills that may influence the learning of letters, numbers, words and comprehension ${ }^{30,31}$.

In order to perform visual perceptual skills (i.e. visual discrimination and visual-spatial skills) children need to have a conceptual understanding of colours, shapes, directionality (up, down, left, right, etc.), distances and sizes, and to be able to identify the finer distinguishing features of different objects ${ }^{32}$. For example, research has shown that children characteristically find it hard to identify letters that have less distinguishing features and that are visually similar to other letters ${ }^{33}$.

The visual processing model is a model explaining how visual perception occurs by means of input, throughput and output. According to the visual processing model, the visual cognitive component of this process involves brain throughput of the sensory information received from the eyes (input). The processed information is sent to other brain regions and body parts for an action response (output) ${ }^{32}$. Visual cognitive processes identify the unique features of the visual information, and they are then analysed so that meaning can be assigned to the unique identifying features of stimuli recorded by the eyes ${ }^{32}$. Difficulties with the visual cognitive processes, such as visual discrimination and visual-spatial skills, negatively impact a child's ability to identify the correct orientation of numbers/letters and contribute to reversals when reading and writing ${ }^{32}$.

It is essential for developers of educational and psychological assessments to meet recommended standards substantiating the reliability and validity of their assessment tools ${ }^{34}$. Thus, studies that explore and demonstrate the reliability of each newly developed assessment are considered critical to establishing the instrument's validity ${ }^{34}$. This study is the first to examine the validity of one such assessment, the Richmond Reversals Rating (RRR) assessment. Spe- 
cifically we examined the known-groups validity and convergent validity in relation to spatial abilities, by means of hypotheses testing $^{33,35,36}$. The RRR assessment was designed based on the principles of visual perception ${ }^{26}$, and constructed from the frame of reference of occupational therapy visual perceptual theory ${ }^{32}$ described above. Subscales within the RRR measure: visual discrimination (ability to discern minor discrepancies in similar forms, shapes, symbols or objects), form constancy (the ability to identify forms, shapes, symbols or objects despite changes in underlying features such as size or shade), figure-ground (the ability to isolate meaningful visual stimuli while ignoring the surrounding stimuli) and visual-spatial skills (recognising and identifying the correct orientation or direction of forms, shapes, symbols or objects) ${ }^{26}$.

Convergent validity testing requires a comparison of a given assessment tool with an existing, well-researched measure of a similar construct ${ }^{36}$. This study compared the RRR assessment tool with an existing measure of the visual-spatial construct, the Spatial Awareness Skills Program Test (SASP) ${ }^{37}$. The SASP is based on the precept that children's spatial awareness and spatial skills increase with development as they age and that inadequate spatial awareness levels negatively impact their ability to perform academic activities $^{37}$. In this regard, the SASP measures children's ability to identify, organise and reproduce detailed spatial geometric forms (rather than letters and numbers) in order to determine whether their measured spatial awareness level is adequate, compared to similarly aged typically developing children ${ }^{37}$.

The known-groups validity explored the RRR assessment's ability to differentiate between school-aged children with below age level, age appropriate and above age level visual-spatial abilities as determined by the SASP. Therefore, the convergent validity results from the RRR assessment were compared to the participants' visual-spatial abilities as measured by construct comparative assessment on the SASP. Hence, the following research Null hypotheses were proposed:

I. The results of the RRR overall scores and participants' visualspatial abilities based on ability level grouping of SASP scores will not demonstrate a statistically significant difference $(p \leq$ $.05)$.

2. The results of the RRR subscale scores and overall scores will not demonstrate a positive correlation to participants' visualspatial abilities based on the SASP scores.

\section{METHOD}

\section{Research design}

The study used a non-experimental, cross sectional research de$\operatorname{sign}^{38}$, with all data being collected from each participant at one time. Its purpose was to explore the relationship between the children's ability to recognise letter and number orientations, as measured by the different RRR subscales, and their levels of visual-spatial abilities and awareness ${ }^{38}$.

\section{Participants}

Seventy-six (36 males and 40 females) Year I- 3 children who ranged in age from 6:02-9:01 years participated in the study (see Table I, below). All the children spoke English as their first language and all attended the same independent primary school located within the north-metropolitan suburbs of Perth, the state capital city of Western Australia. The demographic of the area consists of a mixed Australian, indigenous, and migrant population. There is a low (about $5.5 \%$ ) unemployment and approximately $20 \%$ of parents are professionals. The children were taught to write using Queensland Beginners font. There were no withdrawals from the study. Four participants were left-handed: one in Year I and three in Year 2. In addition, two participants in Year 2 and three in Year 3 were diagnosed with a learning difficulty (dyslexia, dysgraphia or dyscalculia) and one participant in Year I, two participants in Year 2 and one in Year 3 were diagnosed with Attention-Deficit/ Hyperactivity Disorder (ADD/ADHD).

\section{Procedure}

Ethics approval for the research project was obtained from the administrating institution's Human Ethics Subcommittee (HREC I 1444). Non-probability convenience sampling occurred through a local independent school. The principal and teachers gave consent for all parents of Year I-3 students to receive an information letter inviting their child's participation in the study. Only students whose parents signed and returned the consent form participated in the study. Inclusion criteria were: children in Years I-3 who had no diagnosis of hearing impairment requiring alternative communication techniques, no significant un/corrected vision impairment, intellectual impairment, neurological or sensory condition/injury (i.e., traumatic brain injury, cerebral palsy, autism), or psychotic mental disorders. The inclusion criteria were guided by the American Psychiatric Association (APA) diagnostic criteria for specific learning difficulties ${ }^{39}$. Children with the aforementioned conditions were excluded from the study due to the potential confounding effect that their inclusion could potentially have had on the interpretation of the results. All participants provided verbal consent before starting their assessment and were allowed rest breaks. Participation was voluntary and no form of participation remuneration was given to the school, parents or participants. The participants' identities were protected by assigning identification numbers which were used in all electronic records. In addition, the participants' chronological age was not rounded up to the next month when calculated.

Prior to data collection, three children were recruited, using convenience sampling, to participate in a small pilot study in order to refine the data collection process. The data collected from the three pilot participants were not included in the data analysis as these assessments were used for training purposes.

To ensure consistency of administration, one researcher assessed all the participants during school hours, either individually (Year I) or in groups of up to three pupils at a time (Years 2 and 3 ), at times suitable for the class teaching schedules, over a threeweek period half way through the academic year, at the beginning of the third school term. Seventy participants were assessed in a single session lasting approximately 40 minutes in a shared teaching room. The remaining two participants were assessed over two sessions (within one week) as they required extra time to complete the RRR. Assessment times varied slightly according the participants' abilities and level of difficulties. Every effort was made to keep environmental distractions and visual cues for letter and number orientation to a minimum during the assessment process. Participants were asked not to comment verbally on the work so as not to distract other participants, however, they were allowed to ask for clarification if needed.

All participants completed a writing sample first. In this regard, they were asked to write their first names, last names (if they knew how to), the alphabet (preferably using lower case letters) and the numbers 0 to 9 , using a pencil, on standard white paper with blue

Table I: Demographic information of the $\mathbf{7 6}$ participating children

\begin{tabular}{|l|c|c|c|c|c|c|c|c|}
\hline Participants & Grade I & Mean Age & Grade 2 & Mean Age & Grade 3 & Mean Age & Total & Mean Age \\
\hline Male & 17 & $6 y 8 m$ & 7 & $7 y 9 m$ & 12 & $8 y 9 m$ & 36 & $7 y 9 m$ \\
\hline Female & 15 & $6 y 6 m$ & 15 & $7 y 6 m$ & 10 & $8 y 6 m$ & 40 & $7 y 6 m$ \\
\hline Total & 32 & $6 y 7 m$ & 22 & $7 y 8 m$ & 22 & $8 y 7 m$ & 76 & $7 y 6 m$ \\
\hline
\end{tabular}


Table II: Details for RRR subscales content and scoring

\begin{tabular}{|c|l|l|c|}
\hline Subscale & Visual perceptual skill & Content & Maximum score \\
\hline I & Visual discrimination & Upper case letters & 30 \\
\hline II & Visual discrimination & Lower case letters & 20 \\
\hline III & Visual discrimination & Numbers & 37 \\
\hline IV & Spatial orientation & Letters and numbers & 18 \\
\hline V & Form constancy & Letters and numbers & 36 \\
\hline VI & Sequencing & Letters and numbers & 34 \\
\hline VII & Figure ground & Letters in words & 15 \\
\hline VIII & Figure ground & Numbers in calculations & $\mathbf{2 2 6}$ \\
\hline
\end{tabular}

lines. Verbal assistance was provided to sequence the alphabet if necessary. During the writing sample observational notes were taken of the hand preference and any difficulties with pencil grip, reduced hand strength and alphabet writing. The writing sample was followed by the RRR assessment (non-motor), then the Spatial Awareness Skills Program Test (SASP). This allowed the motor tasks to be separated by a non-motor task to provide a rest for the child's hand.

On completion of the assessments parents were provided (upon request) with a confidential summary of their child's assessment results and a presentation of the overall research project results and observations was provided to the school staff.

\section{Richmond Reversal Rating (RRR) Assessment}

The RRR subscale items have been refined based on Rasch measurement analysis and items have been rearranged in hierarchical order to create a linear standardised assessment ${ }^{26,40,41}$. The frame of reference of written letter/number reversals outlined two types of reversal errors ${ }^{14}$. The first is when individual letters or numbers are reversed ${ }^{14}$ and the second is when the order of words or numbers is confused ${ }^{14}$. Seven of the RRR subscales assessed individual reversal errors and the eighth subscale assessed order reversal errors. While, both types of errors are assessed within the RRR, this study specifically focuses on measuring the child's visual cognitive (non-motor) abilities, not visual-motor abilities ${ }^{26}$. The visual cognitive component is the second step (throughput) within the visual perceptual process based on the visual processing model ${ }^{32}$.

All eight subscales of the RRR assessment were completed and during the process reversal recognition abilities of printed numbers, upper case and lower case letters were assessed ${ }^{26}$. Each subscale was designed to assess a different visual perception skill using various combinations of letters and/or numbers (Table II, above).

An RRR subscale accuracy score was calculated for each subscale wherein each item was scored either correct (I) or incorrect (0). The accuracy score was the sum of all reversed letters/numbers or non-reversed letters/numbers correctly identified as such. No score was given for any reversed letters/numbers or non-reversed letters/numbers incorrectly identified. The accuracy scores of the various subscales were added together to calculate the RRR overall accuracy score. Minimal visual-motor abilities are required to complete the RRR assessment as examinees are asked to draw circles to indicate their answers, however, if necessary examinees were allowed to alternatively point to indicate reversed letters and numbers ${ }^{26}$. Spontaneous corrections of first responses were allowed, with examinees erasing the incorrect answer(s).

The RRR assessment uses the Victorian Modern Cursive font ${ }^{\mathrm{a}}$ throughout ${ }^{26}$, with a minor adjustment using Queensland Beginner font for the letters $J$ and $c$, instead of $J$ and $c$.

a The RRR assessment now includes versions in Victorian Modern Cursive, Foundation Cursive Style and Beginner's Print

\section{Spatial Awareness Skills Program Test (SASP)}

Participants' visual spatial ability was assessed using the Spatial Awareness Skills Program Test (SASP), which is suitable to assess children aged 4- 10 years ${ }^{37}$. The SASP measures children's ability to use spatial awareness to near-copy up to 15 line shapes, with increasing level of difficulty ${ }^{37}$. The assessment was administered in accordance with the manual instruction ${ }^{37}$. The SASP has a reported average internal consistency of .76 and an inter-rater reliability of $.96^{37}$. Australian norms were used for scoring the $\mathrm{SASP}^{42}$. The Australian norms provide clearer definitive expected levels of achievement. The norms were developed on $88 \mathrm{I}$ children (446 girls, 435 boys) between 5 and 10 years, the majority in Preprimary through Grade I (675). The Australian norms provided derived scores in terms of percentiles for each age and year group, thus providing clearer expectations than the original age/grade equivalent norms ${ }^{42}$.

\section{Data analysis}

Data collected for each participant were recorded on an Excel spreadsheet and statistically analysed using IBM SPSS Statistics version 24. All assessments were completed in full, with no missing assessment information or scores. The following criteria were used to define the strength of the correlation coefficients: low $=.00$ to .25 , fair $=.26$ to .50 , moderate $=.51$ to .75 , and good $>.75^{36}$. Throughout the report, a $95 \%$ confidence interval and a significance level of $p \geq .05$ ) were used unless specifically stated otherwise.

The SASP manual provides the assessor with age equivalent level scores, in six-month intervals, for the interpretation of the assessment results ${ }^{37}$, however an Australian based study has provided percentiles in age and grade levels which were used in this study ${ }^{42}$. Participant's spatial awareness results were grouped in three ordinal groups by using the participants' percentile score. These ordinal groups were categorised as: below average when the percentile score was below 19 for the year level; average when the percentile was 20 to 82 according to the year level; and above average when the percentile was 83 or more according to the grade level. The three ordinal SASP groups were used to compare and analyse the significance of mean differences and correlation coefficients with the RRR scores.

\section{RESULTS}

Descriptive information for the RRR accuracy scores and SASP scores are provided in Table III on page 27 and indicate a negative skew on the results of the RRR raw scores and an approximate normal distribution of SASP raw scores.

Three ordinal groups created from the SASP results provided known groups' validity, namely below average, average, and above average. In Year I, ten participants were categorised as below average, 17 as average and five as above average; in Year 2, three were below average, I 3 were age average and six were above average; while in Year 3, five participants were classified as below average, 14 as average and three as above average. 
Table III: RRR and SASP raw scores

\begin{tabular}{|c|c|c|c|c|}
\hline RRR overall raw scores & $\begin{array}{c}\text { Year I } \\
\left(n_{1}=32\right)\end{array}$ & $\begin{array}{c}\text { Year } 2 \\
\left(n_{2}=22\right)\end{array}$ & $\begin{array}{c}\text { Year } 3 \\
\left(n_{3}=22\right)\end{array}$ & $\begin{array}{c}\text { Total } \\
(\mathbf{N}=\mathbf{7 6})\end{array}$ \\
\hline Minimum score (minimum 0) & 128 & 164 & 137 & 128 \\
\hline $\begin{array}{l}\text { Maximum score (maximum } \\
\text { 226) }\end{array}$ & 225 & 224 & 224 & 225 \\
\hline Range & 97 & 60 & 87 & 97 \\
\hline Median (Mdn) & 188.50 & 209.0 & 215.0 & 204.0 \\
\hline Means $(\bar{X})$ & 184.91 & 206.95 & 207.45 & 197.82 \\
\hline Standard Deviation (SD) & 25.905 & 14.630 & 21.289 & 24.229 \\
\hline Skewness & -.596 & -1.590 & -2.294 & -1.211 \\
\hline Kurtosis & -.340 & 2.613 & 5.511 & .779 \\
\hline \multicolumn{5}{|l|}{ SASP accuracy scores } \\
\hline Minimum score (minimum 0) & 5 & 5 & 5 & 5 \\
\hline Maximum score (maximum I8) & 12 & 11 & 13 & 13 \\
\hline Range & 7 & 6 & 8 & 8 \\
\hline Median (Mdn) & 7 & 10 & 10 & 9 \\
\hline Means $(\bar{X})$ & 7.66 & 9.27 & 10.23 & 8.87 \\
\hline Standard Deviation (SD) & 1.860 & 1.609 & 1.478 & 1.996 \\
\hline Skewness & .796 & -.867 & .446 & -.052 \\
\hline Kurtosis & -.174 & .595 & -.028 & -.740 \\
\hline
\end{tabular}

Table IV: Spearman's correlation coefficient between RRR and SASP scores

\begin{tabular}{|c|c|c|c|}
\hline & $\begin{array}{l}\text { SASP Grouped Scores } \\
(\mathbf{N}=76)\end{array}$ & $\begin{array}{l}\text { SASP Ranked Percentiles } \\
\qquad(\mathbf{N}=\mathbf{7 6})\end{array}$ & $\begin{array}{l}\text { SASP ranked Raw Scores } \\
\qquad(\mathbf{N}=\mathbf{7 6})\end{array}$ \\
\hline RRR Ranked Overall & $.325 * *(p=.004)$ & $.433 * *(p=.000)$ & $.666 * *(p=.000)$ \\
\hline RRR Ranked Subscale I & $.247 *(p=.031)$ & $.302 * *(p=.008)$ & $.498 * *(p=.000)$ \\
\hline RRR Ranked Subscale II & $.312 * *(p=.006)$ & $.396 * *(p=.000)$ & $.588 * *(p=.000)$ \\
\hline RRR Ranked Subscale III & $.282 *(p=.013)$ & $.394 * *(p=.000)$ & $.578 * *(p=.000)$ \\
\hline RRR Ranked Subscale IV & $.337 * *(p=.003)$ & $.423 * *(p=.000)$ & $.58 I^{* * *}(p=.000)$ \\
\hline RRR Ranked Subscale V & $.077(p=.506)$ & $.162(p=.163)$ & $.281 *(p=.014)$ \\
\hline RRR Ranked Subscale VI & $.252 *(p=.028)$ & $.297 * *(p=.009)$ & $.457^{* *}(p=.000)$ \\
\hline RRR Ranked Subscale VII & $.252 *(p=.028)$ & $.36 I^{* * *}(p=.00 I)$ & $.587^{* *}(p=.000)$ \\
\hline RRR Ranked Subscale VIII & $.313 * *(p=.006)$ & $.44 I^{* * *}(p=.000)$ & $.600 * *(p=.000)$ \\
\hline
\end{tabular}

Due to the ordinal nature of the SASP grouped results, the RRR overall raw scores were analysed using non-parametric statistical analysis. The RRR overall accuracy scores were ranked with mean rankings of $28.14 ; 39.00$, and 50.25 for below average, average and above average groups respectively. The ranked results were compared using the Kruskal-Wallis test to determine the significance of differences between the three independent ordinal SASP groups. This analysis allows indicates that the RRR ranked overall scores were significantly different between the SAPS grouped results, $H(2)=7.956, p=.019$, thus indicating that the RRR has discriminatory ability in visual spatial ability levels. The Jonckheere's Terpstra Test adjusted p-values of the RRR and SASP groups revealed a significant trend in the ranked RRR overall mean scores between participants in the below average and above average groups of $p=.008$, but not between the other groups (below average level and average $p=.119$ and average to above average $p=.147$ ).

\section{Correlations}

The RRR ranked overall scores were correlated with the SASP percentile results using the non-parametric two-tailed Spearman's correlation coefficient. Based on the results from this analysis, a significant fair positive correlation $\left(r_{s}=.433, p=.000\right)$ between the RRR ranked overall scores and the SASP percentile results was found. Table IV above outlines the Spearman's correlation coefficient results between the RRR ranked subscales and the SASP groups.

The RRR ranked overall scores were correlated with the SASP raw scores using the non-parametric two-tailed Spearman's correlation coefficient. Based on the results from this analysis, a significant moderate positive correlation between the RRR ranked overall scores and the SASP raw scores, $\left(r_{s}=.666, p=.000\right)$ was revealed. The Spearman's correlation coefficient results between the RRR ranked subscales and the SASP raw scores demonstrate a significant moderate positive correlation between all the RRR ranked subscale scores and the SASP ranked raw score except for RRR Form Constancy subscale V, which demonstrated a low positive, but significant, correlation coefficient (see Table IV above).

Additional data analysis, using the Kruskal-Wallis test and the Jonckheere's Terpstra test, was performed to determine the significance of the mean results between school years for both 
assessments. In both the RRR and the SASP, there was a significant difference between Year I and Year $2(\operatorname{RRR} p=.003$; SASP $p=.007)$ and between Year I and Year $3(\operatorname{RRR} p=.000$; SASP $p=.000)$, however the significance between Year 2 and Year 3 (RRR $p=1.000$; SASP $p=.515$ ) was not significant.

\section{DISCUSSION}

The visual perceptual frames of reference used by occupational therapists (i.e. visual perceptual skills, including visual-spatial and visual discrimination skills) develop in two ways, namely, through natural maturation and/or learning opportunities ${ }^{32}$. Based on this theory, increasing mean scores as the children progress through the school years are expected on both the RRR and SASP assessments due to their underlying constructs. The results from the study reflect this development with increasing ranked mean scores from Year I to Year 2, and Year 2 to Year 3 for both assessments, with significant differences between Year I and Year 2, and between Year I and Year 3 for both assessments.

The results indicated no significant difference between the ranked RRR and SASP mean scores for participants between Year 2 and Year 3 . As the study's Year 2 participants ranged in age from 7:02 to 8:01 years, these results support previous observations that visual perceptual skills improve with age, but become more stable and reach developmental peak by approximately age 7 years $^{32}$. After age 7 years, visual perceptual skills will increase minimally through the natural maturation process ${ }^{14,32}$, letter reversals observed at later ages are considered a reversal error ${ }^{14}$ and targeted interventions will be necessary to address the deficits ${ }^{14,32}$.

Four Year 3 participants obtained a RRR overall score of $<200$ and, as reported by the parents, three of these four have been diagnosed with a specific learning difficulty and the fourth reportedly experienced increased levels of difficulty in reading, writing, spelling and mathematics. Based on this very small sample, this relationship between the Year 3 results of the RRR assessment and a pre-identified diagnosis of learning difficulty appears promising and would be worthwhile exploring in future studies.

As mentioned, the RRR assesses children's cognitive abilities to recognise letters/numbers written in reversed orientation with minimal visual-motor abilities required during the assessment process. In contrast, the SASP assesses children's spatial awareness but required both visual cognitive (throughput processing) and visualmotor integration (output) skills during the assessment process; the ability to accurately replicate the shape within set boundaries on paper using a pencil influences the assessment results. Thus, the difference between assessing visual processing at primarily a cognitive level as opposed to assessing the visual processing at a visual-motor level may have affected the comparison and interpretation of the results between the RRR and the SASP assessments. This appears to be reflected by the low positive, yet significant, correlation coefficient between the groups as outlined in data analysis and results. In light of this, it may well be that future studies investigate the validity of the RRR assessment using a standardised visual-spatial abilities assessment that measures only the visual-spatial cognitive component for comparison.

The results demonstrate a lower correlation rate between the SASP raw scores and the RRR Sequencing subscale VI. In light of this lower correlation, and the fact that order reversals have been identified as a different type of reversal error ${ }^{14}$, interpretation of the results from this subscale could be considered for separate analysis and interpretation. It is theoretically plausible that interventions used to address difficulties in order reversal errors would be different from interventions used to address difficulties with individual reversal errors, and it would be important for therapist to evaluate and interpret the results for each type of reversal separately and combined when determining the appropriate intervention strategies.

In view of the results and observations made during the assessment process, it is recommended that the time the examinee takes to explore the relationship between RRR items and select, make a choice, and finish the assessment also be recorded. The two participants who required a second session to complete all the RRR subscales both obtained a low RRR overall score of $<160$. Effortful, slow and less automated performance of basic academic activities has been noted as a characteristic of middle school children that have been diagnosed with a learning difficulty ${ }^{43}$. Thus, time taken and level of accuracy-combined scores could be an additional way to interpret the RRR results and to identify children at risk of longterm difficulties.

\section{LIMITATIONS}

The minimum recommended construct validity sample size, using correlation coefficient analysis, is 50 participants ${ }^{44}$. The overall sample size of this study is greater than this minimum, at $N=76$, but the sample size per year group does not meet this minimum sample size criteria, hence no correlation coefficients have been reported by year group. The optimal recommended sample size is 50 participants per subgroup ${ }^{44}$, based on this 150 participants (50 per year or level) would have been more optimal.

Year one students were assessed individually, while Year 2-3 students were assessed in pairs. This may have had a small effect on the outcomes for the older children. As it is usual for children to work in groups at school, this was not seen as a major disadvantage. However, the use of both individual and group assessment should be kept in mind when interpreting the current results. In addition, some bias may have been introduced into the results by maintaining the same order of assessments used in the experiment. This was purposefully done in order to separate the two visual motor component assessments with a motor free assessment, however this ordering effect may have had some minor influence on the assessment outcomes.

The limited timeframe available to approach and recruit a private school to participate in the study made it impractical to be overly selective about the participating school based on the writing font taught by the school. There are a number of differences between the lower case letters used in Australia's Victorian Modern Cursive and Queensland Beginner font, the font taught to the children that participated in this research project. For example: per Victorian Modern font (b. $., l, \sigma, n, r, t$, and $d_{3}$ ) and per Queensland beginner font $(b, i, l, o, p, r, t$, and $z)$.

To reduce the impact these differences in the fonts could have on this study's RRR results, participants were offered and provided with, verbal assistance to name the letter or word when requested by the examinee. Most of the participants noted they were aware of different fonts used to write letters, often commenting that the Victorian Modern font looked like the writing style used by their parents or older siblings. Despite this, the font differences may have had a yet unquantified impact on the results for particular subscales items. Additional analysis to explore the potential impact on each subscale item has yet to be performed.

The above-mentioned limitations could have impacted on the study's results to some extent and, therefore, they should be carefully considered when interpreting the results. In addition this study was non-experimental in nature, which intrinsically limited the interpretation (relationship between variables only, no causeand-effect evidence) that can be associated with the results ${ }^{38}$.

\section{IMPLICATIONS}

The results from this study provide additional evidence for the convergent validity of the RRR scores in relation to young schoolaged children's visual-spatial abilities. For the clinician, the results demonstrated additional evidence for the validity and potential use of the assessment to determine which children experience letter/ number reversal difficulties in order to assist with targeted interventions based on the type of reversal difficulties recorded. For the children struggling with letter/number reversals when reading or writing, the results provide additional support for the ability of the RRR assessment to identify the specific underdeveloped visual 
perceptual skills that are contributing to their reversal difficulties. It is recommended that future researchers focus on using longitudinal, experimental research designs with a sample size that meet the recommended optimal size (by gender, year and diagnosis) to explore and provide increased level of evidence for the assessment's reliability and validity. The RRR participants' results must be observed and analysed over time to refine the interpretation of the assessment results. In addition, further research should focus on the use of the parallel forms that have been created since this project, using Queensland Beginners and Foundation print fonts.

The initial concept of this test came from the researchers clinical observations working in remedial schools in South Africa ${ }^{8,45}$. The concept has been developed, tested and finalised primarily on children in Perth (Australia), however due to the nature of the itinerant population in Perth, South African children are included in the test samples among many other nationalities ${ }^{26}$. Further research on diverse populations in other countries such as South Africa is still required to develop a robust assessment with strong external validity.

\section{REFERENCES}

I. Brooks AD, Berninger VW, Abbott RD. Letter naming and letter writing reversals in children with Dyslexia: Momentary inefficiency in the phonological and orthographic loops of working memory. Developmental Neuropsychology. 201 I; 36(7): 847-68.

2. Frostig M, Horne D. The Frostig programme for the development of visual perception: Teacher's guide. Chicargo, IL: Follett Educational Corporation; 1964

3. Frostig M, Lefever W, Whittlesey JRB. Developmental Test of Visual Perception: Administration and scoring manual. Calif: Consulting psychologists press; 1966.

4. Gordon J, Peterson RL, Boada R, Pennington BF, Treiman R. Statistical Learning, Letter Reversals, and Reading. Scientific Studies of Reading. 20।4; 18(6): 383-94.

5. Heydorn BL. Symbol reversals, reading achievement and handedness in first grade students. Perceptual \& Motor Skills. 1984; 58(2): $589-90$.

6. Liberman IL, Shankweiler D, Orlando C, Harris KS, Berti FB. Letter confusions and reversals of sequence in the beginning reader: Implications for Orton's theory of developmental dyslexia. Cortex. 197I; 7: I27-42.

7. Moyer SB, Newcomer PL. Reversals in reading: diagnosis and remediation. Exceptional children. 1977; 43(7): 424.

8. Richmond J, Taylor M. Visual recognition difficulties: Identifying primary school students' directional confusion in writing letters and numbers. South African Journal of Occupational Therapy. 20 I5; 44(3): 2-6.

9. Richmond JE, Holland K. The relationship between a teacher check list and standardised tests of visual perception skills: A South African perspective. South African Journal of Occupational Therapy. 20 I0; 40(3): 9-16.

10. Terepocki M, Kruk RS, Willows DM. The incidence and nature of letter orientation errors in reading disability. Journal of Learning Disabilities. 2002; 35(3): 2I4-33.

II. Badian NA. Does a visual-orthographic deficit contribute to reading disability? Annals of Dyslexia. 2005; 55(I): 28-52.

12. Boon HC. Relationship of left-right reversals to academic achievement. Perceptual Motor Skills. 1986; 62: 27-33.

13. Cheung P, Poon M, Leung M, Wong R. The Developmental Test of Visual Perception-2 normative study on the visual-perceptual function for children in Hong Kong. Physical \& occupational therapy in pediatrics. 2005; 25(4): 29-43.

14. Lee $S$. A frame of reference for reversal errors in handwriting (A historical review of visual-perceptual theory). School System Special Interest Section Quarterly. 2006; I3(I): I-4.

15. Richmond J, Taylor M, Evans S. Developing bilateral and spatial concepts in primary school-aged children: An empirical evaluation of the Anker Bilateral Spatial System. The Open Journal of Occupational Therapy. 20I4; 2(3): Article 5.

16. Hakim A, Ghorbanibirgani A. Prevalence of Dyslexia among Male Students in Primary Schools and Its Relationship with Obesity and Being Overweight in Ahvaz, Iran. International journal of community based nursing and midwifery. 2015; 3(2): 116

17. Jordan BT, Martin N, Austin JS. Jordan-3: measuring visual reversals in children as symptoms of learning disability and attention-deficit/ hyperactivity disorder. Perceptual and Motor Skills. 20I2; I I5(3): 755.

18. Taylor M, Houghton S, Chapman E. Primitive reflexes and attentiondeficit/hyperactivity disorder: developmental origins of classroom dysfunction. International Journal of Special Education. 2004; I9(I): 23-37.

19. Morgan PL, Farkas G, Tufis PA, Sperling RA. Are Reading and Behavior Problems Risk Factors for Each Other? Journal of Learning Disabilities. 2008; 4I(5): 4I7-36.

20. Daniel SS, Walsh AK, Goldston DB, Arnold EM, Reboussin BA, Wood FB. Suicidality, School Dropout, and Reading Problems Among Adolescents. Journal of Learning Disabilities. 2006; 39(6): 507-I4.

21. Klassen RM, Tze VMC, Hannok W. Internalizing Problems of Adults With Learning Disabilities: A Meta-Analysis. Journal of Learning Disabilities. 20।3; 46(4): 317-27.

22. Nelson JM, Harwood H. Learning Disabilities and Anxiety: A MetaAnalysis. Journal of Learning Disabilities. 20I I; 44(I): 3-I7.

23. Hammerschmidt SL, Sudsawad P. Teachers' survey on problems with handwriting: referral, evaluation, and outcomes. The American journal of occupational therapy : official publication of the American Occupational Therapy Association. 2004; 58(2): 185-92.

24. Gardner MF. Test of Visual Motor Skills Revised. Hydesville, CA Psychological and Educational Publications, Inc; 1996.

25. Kirk SA, Gallagher JJ, Anastasiow NJ. Educating exceptional children. Boston, MA: Houghton Mifflin Company; 2000.

26. Richmond JE. School aged children : Visual perception and reversal recognition of letters and numbers separately and in context. Perth: Edith Cowan University; 2010.

27. Todd VR. Visual information analysis: Frame of reference for visual perception. In: Kramer P, Hinojosa J, editors. Frames of reference for paediatric occupational therapy. Baltimore, MD: Lippincott Williams \& Wilkins; 1999: 205-55.

28. Fisher AG, Murray EA, Bundy AC. Sensory integration theory and practice. Philadelphia: F.A.Davis Company; 1991.

29. Levine KJ. Fine motor dysfunction. Therapeutic strategies in the classroom. Arizona: Therapy Skill Builders (A Division of Psychological Corporation); 199|.

30. Barnes MA, Raghubar KP. Mathematics development and difficulties: The role of visual-spatial perception and other cognitive skills. Pediatric Blood \& Cancer. 2014; 6I(I0): 1729-33.

31. Goldstand S, Koslowe KC, Parush S. Vision, visual-information processing, and academic performance among seventh-grade schoolchildren: A more significant relationship than we thought. American Journal of Occupational Therapy. 2005; 59(4): 377-89.

32. Schneck CM. A frame of reference for visual perception. In: P.Kramer, Hinojosa J, editors. Frames of reference for padiatric occupational therapy. 3rd ed. Philadelphia, PA: Wolters Kluwer / Lippincott Williams \& Wilkins; 2010: 349-89.

33. Bowies RP, Pentimonti JM, Gerde HK, Montroy JJ. Item response analysis of uppercase and lowercase letter naming knowledge. Journal of Psychoeducational Assessment. 20I4; 32(2): I46-56.

34. American Educational Research Association [AERA], American Psychological Association [APA], National Council on Measurement in Education [NCME]. Standards for educational and psychological testing. Washington, DC: American Educational Research Association; 2014.

35. Mokkink LB, Terwee CB, Patrick DL, Alonso J, Stratford PW, Knol $D L$, et al. The COSMIN study reached international consensus on taxonomy, terminology, and definitions of measurement properties for health-related patient-reported outcomes. Journal of Clinical Epidemiology. 2010; 63(7): 737-45.

36. Portney LG, Watkins MP. Foundations of clinical research: Applications to practice. 3 ed. Upper Saddle River, NJ: Prentice-Hall Inc; 2009.

37. Rosner J. Spatial awareness skills program test: Instruction manual. Austin, TX: PRO-ED, Inc.; 1999.

38. DeForge BR. Research Design Principles. In: Salkind NJ, editor. Encyclopedia of Research Design. Thousand Oaks, CA: SAGE Publications, Inc.; 2010: 1253-60.

39. American Psychiatric Publishing, American Psychiatric Association D. S. M. Task Force, American Psychiatric Association. Diagnostic 
and statistical manual of mental disorders: DSM-5. Washington, D.C: American Psychiatric Association; 2013.

40. Richmond JE, Waugh R. Spatial orientation of letters and numbers by young children: A Rasch measurement analysis. AARE Conference:; November 30 to December 3, 2009; Canberra ACT: Australian Association Research in Education; 2009.

4I. Richmond JE, Waugh R. Visual Discrimination of letters in the Alphabet by young children: A Rasch measurement analysis AARE Conference; November 30 to December 3, 2009; Canberra ACT 2009.

42. Rateau F, Laumonier B, Hyndman RJ. Normative Data for the Rosner Test of Visual Analysis Skills on an Australian Population. Optometry \& Vision Science. 2003; 80(6): 43I-6.

43. Graham L, Bellert A, Thomas J, Pegg J. QuickSmart: A Basic Academic Skills Intervention for Middle School Students with Learning Difficulties. Journal of Learning Disabilities. 2007; 40(5): 4I0-9.

44. De Vet HCW, Terwee CB, Mokkink LB, knol DL. Measurement in medicine: A practical guide. Cambridge, United Kingdom: Cambridge University Press; 20II.

45. Richmond J, Holland K. The realtionship between a teacher checklist and standardised tests for visual perception skills: A South African perspective. South African Journal of Occupational Therapy. 20I I; 40(3): 9-16.

\section{Corresponding author}

\section{Dr Janet Richmond}

Email: j.richmond@ecu.edu.au 